\title{
ASYMPTOTIC TOWARDS RAREFACTION WAVE OF THE JIN-XIN RELAXATION MODEL FOR THE $P$ SYSTEM
}

\author{
WEI-CHENG WANG*
}

\begin{abstract}
We study the asymptotic equivalence of the Jin-Xin relaxation model to its formal limit of genuinely nonlinear 2 by 2 conservation laws (isentropic Euler equation in Lagrangian coordinate). We consider the case where the initial data are allowed to have jump discontinuities such that the corresponding solutions to the Euler equation contain centered rarefaction waves. In particular, Riemann data connected by rarefaction curves are included. We show that, as long as the initial data is a small perturbation of a non-vacuum constant state, the solution for the relaxation system exists globally in time and converges, as $\epsilon \rightarrow 0$, to the solution of the corresponding Euler equation uniformly except for an initial layer whose width is essentially of order $O(\epsilon)$.
\end{abstract}

1. Introduction. In this paper, we study the asymptotic behavior of the Jin-Xin model of semilinear hyperbolic system with relaxation:

$$
\begin{array}{ll}
u_{t}+v_{x} & =0 \\
v_{t}+a^{2} u_{x} & =\frac{1}{\epsilon}(\mathcal{F}(u)-v)
\end{array}
$$

as $\epsilon \rightarrow 0$.

The relaxation limit for $2 \times 2$ nonlinear hyperbolic systems was first studied in [7] and further developed in [2]. Distinguished by the special structure of its nonlinear terms, (1.1) was proposed by Jin and Xin in [5] as a relaxational approximation for the quasilinear hyperbolic conservation law

$$
\boldsymbol{u}_{t}+\mathcal{F}(\boldsymbol{u})_{x}=0
$$

This model has an interesting numerical origin. It is used as an artificial approximation for general conservation laws and the authors developed a new class of numerical methods for (1.2) called relaxation schemes based on discretizing (1.1). Owing to the outstanding performance of the relaxation scheme, the hyperbolic system (1.1) has stimulated much research activities.

In the formal limit $\epsilon \rightarrow 0$, we expect the second equation of (1.1) to be well approximated by the local Maxwellian (local equilibrium)

$$
\boldsymbol{v}=\mathcal{F}(\boldsymbol{u})
$$

and the relaxation system reduces to (1.2).

However, the rigorous justification of this asymptotic equivalence is established mostly in the case when (1.2) is a scalar conservation law. For systems of conservation laws, Serre [13] has established a sufficient condition under which one can find an invariant region for (1.1). For examples the Temple system and a system of equations describing motion of elastic material

$$
\partial_{t} u_{1}+\partial_{x} u_{2}=0, \quad \partial_{t} u_{2}+\partial_{x} p\left(u_{1}\right)=0
$$

with constitutive relation satisfying $p^{\prime}>0$ and $s p^{\prime \prime}(s)>0$ for $s \neq 0$. For those systems, the author showed the asymptotic equivalence between (1.1) and (1.2) as $\epsilon \rightarrow 0$. With a slightly different approach, Tzavaras [16], Gosse and Tzavaras [3]

*Department of Mathematics, National Tsing Hua University, Taiwan (wangwc@math.nthu.edu.tw). 
established a strong dissipation estimate for $\boldsymbol{u} \in R^{2}$ with a growth assumption on the flux $f$. The convergence result then follows from the $L^{p}$ theory of compensated compactness.

In this paper, we study the case where the initial data are allowed to have jump discontinuities such that the corresponding solutions of the conservation law contain centered rarefaction waves. To be definite, we restrict ourselves to the isentropic Euler equation in Lagrangian coordinate (also known as the $p$ system):

$$
\boldsymbol{u}=\left(\begin{array}{c}
v \\
u
\end{array}\right), \quad \mathcal{F}(\boldsymbol{u})=\left(\begin{array}{c}
-u \\
p(v)
\end{array}\right), \quad p(v)=v^{-\gamma}, \quad \gamma>1
$$

It is shown that, as long as the initial data is a small perturbation of a non-vacuum constant state, the solution of (1.1) exists globally in time and converges, as $\epsilon \rightarrow 0$, to the solution of (1.2) uniformly except for an initial layer whose width is essentially the order of the mean free path. This is done by approximating the solution of (1.2) with a smooth rarefaction wave followed by a nonlinear stability analysis of the smooth rarefaction wave under discontinuous initial perturbations for (1.1).

Other related works include Natalini [11], who showed the asymptotic equivalence that the solution of the Cauchy problem for (1.1) converges to that of (1.2) strongly in the scalar case. Teng [15] gave an optimal $L^{1}$ error estimate for this asymptotic convergence. An optimal pointwise estimate was derived in Tadmor and Tang [17] based on the optimal $\mathrm{L}^{1}$ estimate and the $\mathrm{Lip}^{+}$estimate. Luo [8] studied the stability of rarefaction wave in the scalar, multidimensional setting of the Jin-Xin model and Luo and Xin [9] showed nonlinear stability of the traveling wave solution in the scalar, multidimensional case. The relaxation approximation was later generalized to Hamilton-Jacobi equation [6] and to curvature dependent front propagation [4].

We choose to present the analysis for Riemann data connected by a rarefaction curve for the $p$ system since

$$
\left(\mathcal{F}^{\prime}(u)\right)^{2}=\left(\begin{array}{cc}
-p^{\prime}(v) & 0 \\
0 & -p^{\prime}(v)
\end{array}\right)
$$

is a positive diagonal matrix, which will simplify the matrix presentation of our energy estimate. Furthermore, the sub-characteristic condition for (1.1) and (1.4) reduces to

$$
-p^{\prime}(v)<a^{2}
$$

We will assume throughout this paper that $a$ is sufficiently large so that (1.6) is trivially satisfied. The result here can be easily extended to the case of two weak centered rarefaction waves of different families, and to general genuinely nonlinear 2 by 2 conservation laws equipped with a convex entropy. We omit these cases to make the presentation brief and more readable.

The rest of the paper is organized as follows: In section 2, we construct the smooth approximate solution and list some preliminary estimates about it. We then state the main theorem (Theorem 2.1). In sections 3, we proceed to prove the main theorem by treating the Riemann initial data as a discontinuous perturbation of the smooth approximation. We then proceed by a piecewise $H^{1}$ estimate on the error. To this end, we first study how the initial jump propagates and decays along the characteristics in (1.1). We then finish the proof by piecewise energy estimate and the Sobolev inequality. 
2. Smooth approximations. We first rewrite (1.1) in a simpler form as

$$
\begin{gathered}
\boldsymbol{F}_{t}^{\epsilon}+\boldsymbol{V} \boldsymbol{F}_{x}^{\epsilon}=\frac{1}{\epsilon} \mathcal{N}\left(\boldsymbol{F}^{\epsilon}\right) \quad t \geq 0, \quad x \in R \\
\boldsymbol{F}^{\epsilon}(x, 0)= \begin{cases}\boldsymbol{F}_{r} & \text { if } x>0 \\
\boldsymbol{F}_{l} & \text { if } x<0\end{cases}
\end{gathered}
$$

and the scaled version of $(2.1)$ :

$$
\begin{gathered}
\boldsymbol{F}_{t}+V \boldsymbol{F}_{x}=\mathcal{N}(\boldsymbol{F}) \quad t \geq 0, \quad x \in R \\
\boldsymbol{F}(x, 0)= \begin{cases}\boldsymbol{F}_{r} & \text { if } x>0 \\
\boldsymbol{F}_{l} & \text { if } x<0\end{cases}
\end{gathered}
$$

where

$$
\boldsymbol{F}=\left(\begin{array}{l}
\boldsymbol{u} \\
\boldsymbol{v}
\end{array}\right), \quad \mathcal{N}(\boldsymbol{F})=\left(\begin{array}{c}
0 \\
\mathcal{F}(\boldsymbol{u})-\boldsymbol{v}
\end{array}\right), \quad \boldsymbol{V}=\left(\begin{array}{cc}
0 & I_{2} \\
a^{2} I_{2} & 0
\end{array}\right)
$$

and $\boldsymbol{F}_{r}$ and $\boldsymbol{F}_{l}$ are both in local equilibrium (1.3).

It is clear that $\boldsymbol{F}(x, t)$ is a solution of (2.2) if and only if

$$
\boldsymbol{F}^{\epsilon}(x, t) \stackrel{\text { def }}{=} \boldsymbol{F}(x / \epsilon, t / \epsilon)
$$

is a solution of (2.1).

We further assume that the Riemann Cauchy data $\left(\boldsymbol{F}_{l}, \boldsymbol{F}_{r}\right)$ for (2.2) corresponds to Riemann Cauchy data $\left(\left(v_{l}, u_{l},-u_{l}, p\left(v_{l}\right)\right)^{T},\left(v_{r}, u_{r},-u_{r}, p\left(v_{r}\right)\right)^{T}\right)$ for (1.1) such that $\left(v_{l}, u_{l}\right)$ and $\left(v_{r}, u_{r}\right)$ are connected by a rarefaction curve. Thus the solution of (1.2) is a self similar centered rarefaction wave:

$$
(v, u)(x, t)=\left(v^{c r}, u^{c r}\right)(x / t)
$$

(For an introduction on Cauchy problems with Riemann initial data and rarefaction curves, see [14].)

We want to study the time asymptotic/small mean free path limit of $(2.2) /(2.1)$. We will show that if $\left|u_{l}-u_{r}\right|$ are small enough and (1.6) holds, (2.2) has a unique global in time solution and this solution is asymptotically equivalent to a self similar function $\boldsymbol{F}^{c r}$ :

$$
\lim _{t \rightarrow \infty} \sup _{x \in R}\left|\boldsymbol{F}(x, t)-\boldsymbol{F}^{c r}(x / t)\right|=0,
$$

where $\boldsymbol{F}^{c r}$ is obtained by imposing local equilibrium (1.3) together with (2.5):

$$
\boldsymbol{F}^{c r}=\left(\begin{array}{c}
\boldsymbol{u}^{c r} \\
\mathcal{F}\left(\boldsymbol{u}^{c r}\right)
\end{array}\right)
$$

In view of (2.4), we see that (2.1) has a unique solution satisfying

$$
\lim _{\epsilon \rightarrow 0} \sup _{x \in R, t \geq \epsilon^{\delta}}\left|\boldsymbol{F}^{\epsilon}(x, t)-\boldsymbol{F}^{c r}(x / t)\right|=0
$$

for any $\delta<1$ (one can replace $\epsilon^{\delta}$ by any $g(\epsilon)$ such that $\epsilon=o(g(\epsilon))$ as $\epsilon \rightarrow 0$ ).

In order to prove (2.6), we will construct $\boldsymbol{G}(x, t)$, a smooth approximation of $\boldsymbol{F}^{c r}$ and show that both $\boldsymbol{F}-\boldsymbol{G}$ and $\boldsymbol{F}^{c r}-\boldsymbol{G}$ are asymptotically equivalent (Lemma 2.3 (b) and (2.20) below). 
We proceed by constructing $\boldsymbol{G}$ as follows:

Let $\tilde{w}(x, t)$ be the solution of the following Cauchy problem:

$$
\begin{aligned}
& \tilde{w}_{t}+\tilde{w} \tilde{w}_{x}=0 \\
& \tilde{w}(x, 0)=\frac{1}{2}\left\{\left(w_{r}+w_{l}\right)+\left(w_{r}-w_{l}\right) \tanh x\right\}
\end{aligned}
$$

LEMMA 2.1. [10] Suppose $w_{r}>w_{l}$, then (2.9) has a unique global smooth solution satisfying

(a) $w_{l}<\tilde{w}(x, t)<w_{r}, \tilde{w}_{x}(x, t)>0$ for $t \geq 0, x \in R$.

(b) For any $p \in[1, \infty]$, there is a positive constant $C$ such that for $t \geq 0$,

$$
\begin{aligned}
& \left\|\tilde{w}_{x}(t)\right\|_{L^{p}} \leq C \min \left(\left|w_{r}-w_{l}\right|,\left|w_{r}-w_{l}\right|(1+t)^{-1+\frac{1}{p}}\right), \\
& \left\|\tilde{w}_{x x}(t)\right\|_{L^{p}},\left\|\tilde{w}_{x x x}(t)\right\|_{L^{p}} \leq C \min \left(\left|w_{r}-w_{l}\right|,(1+t)^{-1}\right) .
\end{aligned}
$$

(c) $\lim _{t \rightarrow \infty} \sup _{x \in R}\left|\tilde{w}(x, t)-w^{c r}(x / t)\right|=0$.

Since the rarefaction wave solution written in the Riemann invariant coordinate reduces to a rarefaction wave for the Burgers' equation up to a nonlinear change of variables, we can thus construct the corresponding solutions of the Euler equation by inverting this change of variables. The corresponding solution of (1.2), (3.34), $(\tilde{v}, \tilde{u})$ satisfies

LEMMA 2.2. For each $\left(v_{l}, u_{l}\right)$ satisfying the sub-characteristic condition (1.6) there exists $\delta_{0}>0$ such that if $\left(v_{r}, u_{r}\right)$ can be connected to $\left(v_{l}, u_{l}\right)$ by a centered rarefaction wave $\left(v^{c r}, u^{c r}\right)$ and $\left|v_{l}-v_{r}\right|<\delta_{0}$, then the corresponding smooth approximation $(\tilde{v}, \tilde{u})$ satisfies the following:

(a) $(\tilde{v}, \tilde{u})$ is a smooth global solution in time of

$$
\begin{aligned}
& \tilde{v}_{t}-\tilde{u}_{x}=0 \\
& \tilde{u}_{t}+p(\tilde{u})_{x}=0
\end{aligned} .
$$

(b) For any $p \in[1, \infty]$, there is a positive constant $C$ such that for $t \geq 0$,

$$
\begin{aligned}
& \left\|\tilde{u}_{x}(t), \tilde{u}_{t}(t)\right\|_{L^{p}} \leq C \min \left(\left|u_{r}-u_{l}\right|,\left|u_{r}-u_{l}\right|^{1 / p}(1+t)^{-1+\frac{1}{p}}\right), \\
& \left\|\tilde{u}_{x x}(t), \tilde{u}_{t x}(t), \tilde{u}_{x x x}(t), \tilde{u}_{t x x}(t)\right\|_{L^{p}} \leq C \min \left(\left|u_{r}-u_{l}\right|,(1+t)^{-1}\right) .
\end{aligned}
$$

(c) $\lim _{t \rightarrow \infty} \sup _{x \in R}\left|(\tilde{v}, \tilde{u})(x, t)-\left(v^{c r}, u^{c r}\right)(x / t)\right|=0$.

Let us denote the zeroth order approximation of $\boldsymbol{F}^{c r}$ by

$$
\boldsymbol{F}^{(0)}=\left(\begin{array}{c}
\boldsymbol{u}^{(0)} \\
\mathcal{F}\left(\boldsymbol{u}^{(0)}\right)
\end{array}\right), \quad \boldsymbol{u}^{(0)}=\left(\begin{array}{c}
\tilde{v} \\
\tilde{u}
\end{array}\right)
$$

and following the Chapman-Enskog expansion procedure outlined in [1], we get the first order correction

$$
\boldsymbol{F}^{(1)}=\left(\begin{array}{c}
0 \\
\boldsymbol{v}^{(1)}
\end{array}\right), \quad \boldsymbol{v}^{(1)}=-\left(a^{2}-\mathcal{F}^{\prime}\left(\boldsymbol{u}^{(0)}\right)^{2}\right) \boldsymbol{u}_{x}^{(0)}
$$


We then construct $\boldsymbol{G}$, the smooth approximation of $\boldsymbol{F}^{c r}$, by

$$
G=F^{(0)}+F^{(1)}
$$

Thus $\boldsymbol{G}$ satisfies the following equation

$$
\boldsymbol{G}_{t}+\boldsymbol{V} \boldsymbol{G}_{x}-\mathcal{N}(\boldsymbol{G})=\boldsymbol{F}_{t}^{(1)}+\boldsymbol{V} \boldsymbol{F}_{x}^{(1)}
$$

and we have the corresponding estimates for $G$ :

LEMMA 2.3. Under the same assumption in lemma 2.2, the smooth approximation $G$ satisfies (2.16) and

(a) $\mathcal{N}(\boldsymbol{G})=-\boldsymbol{v}^{(1)}$

(b) $\lim _{t \rightarrow \infty} \sup _{x \in R}\left|\boldsymbol{G}(x, t)-\boldsymbol{F}^{c r}(x / t)\right|=0$.

To show the asymptotic equivalence of $\boldsymbol{F}$ and $\boldsymbol{G}$, we let $\boldsymbol{f}=\boldsymbol{F}-\boldsymbol{G}$. The equation satisfied by $f$ reads

$$
\begin{aligned}
& \boldsymbol{f}_{t}+\boldsymbol{V} \boldsymbol{f}_{x}=\mathcal{N}(\boldsymbol{F})-\mathcal{N}(\boldsymbol{G})-\left(\boldsymbol{F}_{t}^{(1)}+\boldsymbol{V} \boldsymbol{F}_{x}^{(1)}\right) \\
&=\left(\begin{array}{c}
0 \\
\boldsymbol{\nu}-\boldsymbol{A} \boldsymbol{\mu}
\end{array}\right)+O\left(|\boldsymbol{\mu}|^{3}+\left|\boldsymbol{v}_{t}^{(1)}\right|+\left|\boldsymbol{v}_{x}^{(1)}\right|\right) \\
& \boldsymbol{f}(x, 0)=\boldsymbol{F}(x, 0)-\boldsymbol{G}(x, 0)
\end{aligned}
$$

where $\boldsymbol{A}=\mathcal{F}^{\prime}\left(\boldsymbol{u}^{(0)}+\boldsymbol{\mu} / 2\right)$.

(2.17), like (1.1), is a semilinear hyperbolic system. The discontinuities propagate along $x=a t$ and $x=-a t$. Thus we adopt the piecewise energy estimate. We introduce the following notations: Denote by $\Omega_{k}, k=1,2,3$ the regions separated by $x=a t$ and $x=-a t$ in the upper half plane $t>0, \Omega_{k}^{s}=\Omega_{k} \cap\{t=s\}$ and for any interval $I \subset R, H^{1}(I)$ the usual Sobolev space with norm $\|\cdot\|_{1}=\left(\|\cdot\|_{L^{2}(I)}+\| \frac{\partial}{\partial x}\right.$. $\left.\|_{L^{2}(I)}\right)^{1 / 2}$.

Now we define the appropriate function space on which we will be working:

$$
\begin{aligned}
X(0, T)=\{\boldsymbol{h}: R \times & {[0, T] \mapsto R^{4} \mid \boldsymbol{h} \in C^{0}([0, T),} \\
& \left.\left.H^{1}\left(\Omega_{k}^{s}\right)\right) \cap C^{1}\left([0, T), L^{2}\left(\Omega_{k}^{s}\right)\right) \cap C^{2}\left(\bar{\Omega}_{k}\right), k=1,2,3\right\}
\end{aligned}
$$

For $h \in X(0, T)$, we define

$$
\begin{aligned}
& \| \boldsymbol{h}(\cdot, t) \mathbb{H}^{2}=f|\boldsymbol{h}(x, t)|^{2} d x \\
& =\int_{-\infty}^{-t}|\boldsymbol{h}(x, t)|^{2} d x+\int_{-t}^{t}|\boldsymbol{h}(x, t)|^{2} d x+\int_{t}^{\infty}|\boldsymbol{h}(x, t)|^{2} d x
\end{aligned}
$$

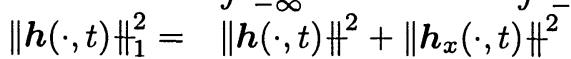

$$
\begin{aligned}
& {[\boldsymbol{h}]^{+}(t)=\boldsymbol{h}\left(t^{+}, t\right)-\boldsymbol{h}\left(t^{-}, t\right) \quad\langle\boldsymbol{h}\rangle^{+}(t)=\frac{1}{2}\left(\boldsymbol{h}\left(t^{+}, t\right)+\boldsymbol{h}\left(t^{-}, t\right)\right)} \\
& {[\boldsymbol{h}]^{-}(t)=\boldsymbol{h}\left(-t^{+}, t\right)-\boldsymbol{h}\left(-t^{-}, t\right) \quad\langle\boldsymbol{h}\rangle^{-}(t)=\frac{1}{2}\left(\boldsymbol{h}\left(-t^{+}, t\right)+\boldsymbol{h}\left(-t^{-}, t\right)\right) \text {. }}
\end{aligned}
$$

We want to show

$$
\lim _{t \rightarrow \infty} \sup _{x \in R}|\boldsymbol{F}(x, t)-\boldsymbol{G}(x, t)|=0 .
$$


A key observation is the following: The a priori bound on $\sup _{t>0}\|f\|_{L^{\infty}(t)}$ implies exponential decay in time of the jumps (see Lemma 3.1 below). Thus in view of the Sobolev inequality

$$
\|\boldsymbol{f}\|_{L^{\infty}}^{2}(t) \leq C\left(\|\boldsymbol{f}\| \| \boldsymbol{f}_{x} H(t)+[\boldsymbol{f}]_{+}^{2}(t)+[\boldsymbol{f}]_{-}^{2}(t)\right),
$$

our task remains to estimate $\|\boldsymbol{f}\|_{1}$ (Theorem 2.1 below). During this process, terms involving line integrals of jumps across the discontinuities appear naturally. Therefor the exponential decay in time of the jumps implies the a priori bound on $\sup _{t \geq 0} \| f \mathbb{H}_{1}^{2}(t)$ (Lemma 3.3 and on). We close this bootstrapping argument by the local existence theorem (Theorem 2.2 below) to extend $f$ in $X(0, T+\Delta t)$ and conclude that $T=\infty$ (global in time existence).

THEOREM 2.1 (A priori estimate). There exists positive constants $\epsilon_{1}$ and $C_{1}$ such that if $f \in X(0, T)$ is the solution of (2.17) in $0 \leq t \leq T$ for some $T>0$ and

$$
\sup _{0 \leq t \leq T} \| \boldsymbol{f}(t) \#_{1}+\left|u_{r}-u_{l}\right|<\epsilon_{1}
$$

then

$$
\sup _{0 \leq t \leq T}\left\|\boldsymbol{f}(t) H_{1}^{2}+\int_{0}^{T}\right\| \boldsymbol{f}_{x}(\tau) H^{2} \leq C_{1}\left(\| \boldsymbol{f}(0) H_{1}^{2}+\left|u_{r}-u_{l}\right|^{1 / 6}\right) .
$$

The proof of Theorem 2.1 will occupy Section 3.

THEOREM 2.2 (Local existence). Let $T \geq 0$ and $g \in X(0, T)$ be a solution to (2.17) for $0 \leq t \leq T$. Consider the initial value problem to (2.17) with the initial datum

$$
\boldsymbol{f}(T, x)=\boldsymbol{g}_{T}(x) \stackrel{\text { def }}{=} \boldsymbol{g}(T, x) .
$$

Then for any $M>0$, there exists a positive constant $\Delta t$ depending only on $M$ and $\sup _{x, t}|\boldsymbol{G}(x, t)|$ such that if

$$
\left\|\boldsymbol{g}_{T}\right\|_{C^{0}(-\infty,-a T]}+\left\|\boldsymbol{g}_{T}\right\|_{C^{0}[-a T, a T]}+\left\|\boldsymbol{g}_{T}\right\|_{C^{0}[a T, \infty)}<M
$$

Then (2.17) together with (2.23) has a unique solution $f \in X(T, T+\Delta t)$ satisfying

$$
\sup _{T \leq t \leq T+\Delta t}\left(\|f(t)\|_{C^{0}(-\infty,-a t]}+\|f(t)\|_{C^{0}[-a t, a t]}+\|f(t)\|_{C^{0}[a t, \infty)}\right)<2 M .
$$

As a consequence, $\boldsymbol{g}$ can be extended to $X(0, T+\Delta t)$.

The proof of Theorem 2.2 is standard, see [12] for the existence and uniqueness in the piecewise $C^{0}$ function class. The piecewise $C^{2}$ regularity is a direct consequence of the special structure of the nonlinearity (being the lower order term in (2.17)) and the $C^{2}$ regularity of the source term $G$.

¿From Theorem 2.1 and Theorem 2.2, we have the following

Corollary 2.1. For each $\left(v_{l}, u_{l}\right)$, with $v_{l}>0$ there exists $\epsilon_{0}$ and $C_{0}$ such that if $\left(v_{r}, u_{r}\right)$ can be connected to $\left(v_{l}, u_{l}\right)$ by a centered rarefaction wave and $\| \boldsymbol{f}(0) \mathrm{H}_{1}+$ $\left|u_{r}-u_{l}\right|<\epsilon_{0}$, then (2.17) has a unique solution $f \in X(0, \infty)$ satisfying

$$
\sup _{t \geq 0}\left\|f(t) H_{1}^{2}+\int_{0}^{\infty}\right\| f_{x}(\tau) \#^{2} d \tau \leq C_{0}\left(\| f(0) H_{1}^{2}+\left|u_{r}-u_{l}\right|^{1 / 6}\right) .
$$


With Corollary 2.1, Lemma 3.1 below and the Sobolev inequality (2.21), it is easy to see that

$$
\int_{0}^{\infty}\left\|f_{x} \#^{2}(\tau)+\left|\frac{d}{d \tau} \| f_{x} \#^{2}\right|(\tau)<\infty\right.
$$

and consequently (2.20) holds.

3. A priori estimates. We first introduce here the entropy extension for the Jin-Xin system:

Proposition 3.1. Let $(\phi(\boldsymbol{u}), \psi(\boldsymbol{u}))$ be a pair of entropy-flux functions of (1.2) with $\phi$ convex, then we can derive the corresponding entropy-flux pair $(\Phi, \Psi)$ for the system (1.1) from the following wave equation with Cauchy data

$$
\begin{aligned}
& \Phi_{\boldsymbol{u}}=\Psi_{\boldsymbol{v}} \\
& \Psi_{\boldsymbol{u}}=a^{2} \Phi_{\boldsymbol{v}} \\
& \Phi_{(\boldsymbol{u}, \mathcal{F}(\boldsymbol{u}))=\phi(\boldsymbol{u})} \\
& \Phi_{\boldsymbol{v}}(\boldsymbol{u}, \mathcal{F}(\boldsymbol{u}))=0
\end{aligned}
$$

The solution $(\Phi, \Psi)$ exists in a neighborhood of the equilibrium $\boldsymbol{v}=\mathcal{F}(\boldsymbol{u})$ and there it satisfies

(a) $\Phi$ is convex if a is sufficiently large.

(b) $\Psi(\boldsymbol{u}, \mathcal{F}(\boldsymbol{u}))=\psi(\boldsymbol{u})$.

(c) $\Phi_{t}(\boldsymbol{u}, \boldsymbol{v})+\Psi_{x}(\boldsymbol{u}, \boldsymbol{v})=-\frac{1}{\epsilon} \Phi_{\boldsymbol{v}}(\boldsymbol{u}, \boldsymbol{v}) \cdot(\boldsymbol{v}-\mathcal{F}(\boldsymbol{u})) \leq 0$ for any smooth solution of (1.1).

We remark here that the solution in general exists only in a neighborhood of the equilibrium $\boldsymbol{v}=\mathcal{F}(\boldsymbol{u})$ and is given explicitly by

$$
\begin{aligned}
& \Phi(\boldsymbol{u}, \boldsymbol{v})=h^{+}(\boldsymbol{v}+a \boldsymbol{u})+h^{-}(\boldsymbol{v}-a \boldsymbol{u}) \\
& \Psi(\boldsymbol{u}, \boldsymbol{v})=a\left(h^{+}(\boldsymbol{v}+a \boldsymbol{u})-h^{-}(\boldsymbol{v}-a \boldsymbol{u})\right)
\end{aligned}
$$

where $h^{ \pm}(\cdot)$ is given implicitly by

$$
h^{ \pm}(\mathcal{F}(\boldsymbol{u}) \pm a \boldsymbol{u})=\frac{1}{2}\left(\phi(\boldsymbol{u}) \pm \frac{\psi(\boldsymbol{u})}{a}\right) .
$$

For the $p$ system

$$
\boldsymbol{u}=\left(\begin{array}{l}
v \\
u
\end{array}\right), \quad \mathcal{F}(\boldsymbol{u})=\left(\begin{array}{l}
-u \\
p(v)
\end{array}\right)
$$

we take $\phi$ the mechanical energy

$$
\phi(\boldsymbol{u})=\frac{1}{2} u^{2}-\int^{v} p(s) d s,
$$

and use the corresponding derived entropy $\Phi(\boldsymbol{u}, \boldsymbol{v})$ in the main energy estimate to follow.

In order to perform the energy estimate, it is important to study how the singularity propagates. (1.1) is a semilinear hyperbolic system, the jump discontinuity in of the initial data propagate along the characteristic curves and, due to the relaxation 
effect, decays exponentially in time. To show this, we write (1.1) in diagonal form in the characteristic variables $\boldsymbol{w}^{ \pm}=\boldsymbol{v} \pm a \boldsymbol{u}$,

$$
\begin{aligned}
& \boldsymbol{w}_{t}^{+}+a \boldsymbol{w}_{x}^{+}=\frac{1}{2}(\mathcal{F}(\boldsymbol{u})-\boldsymbol{v}) \\
& \boldsymbol{w}_{t}^{-}-a \boldsymbol{w}_{x}^{-}=\frac{1}{2}(\mathcal{F}(\boldsymbol{u})-\boldsymbol{v})
\end{aligned}
$$

We denote by $[\cdot]^{ \pm}$the jumps along the characteristic line $d x / d t= \pm a$. Taking the jump in the ' + ' family on both sides of the first equation in (3.31), we have

$$
\left[\boldsymbol{w}_{t}^{+}+a \boldsymbol{w}_{x}^{+}\right]^{+}=\frac{1}{2}\left(\left[\mathcal{F}\left(\frac{\boldsymbol{w}^{+}-\boldsymbol{w}_{r}^{-}}{2 a}\right)\right]^{+}-\left[\boldsymbol{w}^{+}\right]^{+}\right) \text {. }
$$

where $\boldsymbol{w}_{r}^{-}=\boldsymbol{v}_{r}-a \boldsymbol{u}_{r}$ is the corresponding Riemann data on $x>0$. Since $\boldsymbol{F}$ has continuous first derivatives up to the boundary on either side of the jumps, we can interchange the tangential derivative with the jump to get an ODE along the characteristics

$$
\frac{d}{d t}\left[\boldsymbol{w}^{+}\right]^{+}=\frac{1}{2}\left(\left[\mathcal{F}\left(\frac{\boldsymbol{w}^{+}-\boldsymbol{w}_{r}^{-}}{2 a}\right)\right]^{+}-\left[\boldsymbol{w}^{+}\right]^{+}\right) .
$$

By standard ODE theory, $\left[\boldsymbol{w}^{+}\right]^{+}(t)$ decays exponentially in $t$ together with $\left[\boldsymbol{w}_{x}^{ \pm}\right]^{+}(t)$, provided $a$ is sufficiently large. The same applies to $\left[\boldsymbol{w}^{-}, \boldsymbol{w}_{x}^{ \pm}\right]^{-}(t)$. We therefore have the following

LEMMA 3.1. Let $\alpha=\left|u_{r}-u_{l}\right|$ and $E=$ $\sup _{0 \leq t \leq T}\left(\| \boldsymbol{f} H_{1}(t)+\left|[\boldsymbol{f}]^{+}(t)\right|+\left|[\boldsymbol{f}]^{-}(t)\right|\right)$, then there exist positive constants $\epsilon_{1}, C$ and $C_{1}$ such that if $0 \leq t \leq T, E \leq \epsilon_{1}$ and a sufficiently large, we have

$$
\left|[\boldsymbol{f}]^{ \pm}(t)\right|+\left|\left[\boldsymbol{f}_{x}\right]^{ \pm}(t)\right| \leq C \alpha e^{-C_{1} t}
$$

We now proceed with the main energy estimate.

Let $\alpha$ and $E$ be defined as in Lemma 3.1, we have

LEMMA 3.2. There exist positive constants $\epsilon_{2}$ and $C$ such that if $0 \leq t \leq T$ and $E \leq \epsilon_{2}$, then

$$
\begin{aligned}
& \|\boldsymbol{f}(t)\|^{2}+\int_{0}^{t}\|\boldsymbol{\nu}-\boldsymbol{A} \boldsymbol{\mu}\|^{2} d \tau \\
\leq & \|\boldsymbol{f}(0)\|^{2}+C \int_{0}^{t} f\left(\left|\boldsymbol{v}^{(1)}\right|^{4}+\left|\boldsymbol{v}_{x}^{(1)}\right|^{2}+|\boldsymbol{f}|^{2}\left|\boldsymbol{v}^{(1)}\right|+\left|\boldsymbol{f} \| \boldsymbol{v}_{x}^{(1)}\right|+|\boldsymbol{f}|^{6}\right) d x d \tau+C \alpha
\end{aligned}
$$

Proof. We introduce here the quadratic part of the increment of the derived entropy-flux pair and corresponding partial derivatives for the purpose of $L^{2}$ estimate:

$$
\begin{array}{ll}
\mathcal{E}(\boldsymbol{f}, \boldsymbol{G}) & \stackrel{\text { def }}{=} \Phi(\boldsymbol{G}+\boldsymbol{f})-\Phi(\boldsymbol{G})-\Phi^{\prime}(\boldsymbol{G}) \boldsymbol{f} \\
\mathcal{E}_{\boldsymbol{f}}(\boldsymbol{f}, \boldsymbol{G}) & =\Phi^{\prime}(\boldsymbol{G}+\boldsymbol{f})-\Phi^{\prime}(\boldsymbol{G}) \\
\mathcal{E}_{\boldsymbol{G}}(\boldsymbol{f}, \boldsymbol{G}) & =\Phi^{\prime}(\boldsymbol{G}+\boldsymbol{f})-\Phi^{\prime}(\boldsymbol{G})-\boldsymbol{f}^{T} \Phi^{\prime \prime}(\boldsymbol{G})
\end{array}
$$

and

$$
\begin{array}{lll}
\mathcal{J}(\boldsymbol{f}, \boldsymbol{G}) & \stackrel{\text { def }}{=} \Psi(\boldsymbol{G}+\boldsymbol{f})-\Psi(\boldsymbol{G})-\Psi^{\prime}(\boldsymbol{G}) \boldsymbol{f} \\
\mathcal{J}_{\boldsymbol{f}}(\boldsymbol{f}, \boldsymbol{G}) & =\Psi^{\prime}(\boldsymbol{G}+\boldsymbol{f})-\Psi^{\prime}(\boldsymbol{G}) \\
\mathcal{J}_{\boldsymbol{G}}(\boldsymbol{f}, \boldsymbol{G}) & =\Psi^{\prime}(\boldsymbol{G}+\boldsymbol{f})-\Psi^{\prime}(\boldsymbol{G})-\boldsymbol{f}^{T} \Psi^{\prime \prime}(\boldsymbol{G})
\end{array}
$$


It is easy to see that, for $E<\epsilon_{2}$, we have

$$
\begin{aligned}
& \nu|f|^{2} \leq \mathcal{E}(\boldsymbol{f}, \boldsymbol{G}) \leq C|\boldsymbol{f}|^{2} \\
& \mathcal{E}_{\boldsymbol{f}}(\boldsymbol{f}, \boldsymbol{G}) \leq C|\boldsymbol{f}| \\
& \mathcal{E}_{\boldsymbol{G}}(\boldsymbol{f}, \boldsymbol{G}) \leq C|\boldsymbol{f}|^{2}
\end{aligned}
$$

Next we multiply $(2.17)$ by $\mathcal{E}_{\boldsymbol{f}}(\boldsymbol{f}, \boldsymbol{G})$,

$$
\mathcal{E}_{t}+\mathcal{J}_{x}=\mathcal{E}_{\boldsymbol{G}}\left(\boldsymbol{G}_{t}+\boldsymbol{V} \boldsymbol{G}_{x}\right)+\mathcal{E}_{\boldsymbol{f}}(\mathcal{N}(\boldsymbol{G}+\boldsymbol{f})-\mathcal{N}(\boldsymbol{f}))-\mathcal{E}_{\boldsymbol{f}}\left(\boldsymbol{F}_{t}^{(1)}+\boldsymbol{V} \boldsymbol{F}_{f}^{(1)}\right)
$$

where we have used $\Phi^{\prime} V=\Psi^{\prime}$.

Next we calculate each term on the right hand side of (3.39):

$$
\begin{gathered}
\boldsymbol{G}_{t}+\boldsymbol{V} \boldsymbol{G}_{x}=\mathcal{N}(\boldsymbol{G})+\boldsymbol{F}_{t}^{(1)}+\boldsymbol{V} \boldsymbol{F}_{x}^{(1)}=O\left(\boldsymbol{v}^{(1)}, \boldsymbol{v}_{t}^{(1)}, \boldsymbol{v}_{x}^{(1)}\right) \\
\mathcal{N}(\boldsymbol{G}+\boldsymbol{f})-\mathcal{N}(\boldsymbol{f})=\left(\begin{array}{c}
0 \\
\boldsymbol{\nu}-\boldsymbol{A} \boldsymbol{\mu}+O\left(|\boldsymbol{\mu}|^{3}\right)
\end{array}\right)
\end{gathered}
$$

and by expanding the $\mathcal{E}_{\boldsymbol{f}}$ around $\boldsymbol{G}+\boldsymbol{f} / 2$, we have

$$
\mathcal{E}_{\boldsymbol{\mu}}=\frac{\left(a^{2} \boldsymbol{\mu}-\boldsymbol{A} \boldsymbol{\nu}\right)^{T} \phi^{\prime \prime}}{\left(a^{2}-\boldsymbol{A}^{2}\right)}+O\left(\left|\boldsymbol{v}^{(1)}\right|^{2}+|\boldsymbol{f}|^{2}\right)
$$

$$
\mathcal{E}_{\boldsymbol{\nu}}=\frac{(\boldsymbol{\nu}-\boldsymbol{A} \boldsymbol{\mu})^{T} \phi^{\prime \prime}}{\left(a^{2}-\boldsymbol{A}^{2}\right)}+O\left(\left|\boldsymbol{v}^{(1)}\right||\boldsymbol{f}|+|\boldsymbol{f} \| \boldsymbol{\nu}-\boldsymbol{A} \boldsymbol{\mu}|\right)+O\left(\left|\boldsymbol{v}^{(1)}\right|^{3}+|\boldsymbol{f}|^{3}\right)
$$

where both $\phi^{\prime \prime}$ and $\boldsymbol{A}$ are evaluated at $\boldsymbol{u}_{0}+\boldsymbol{\mu} / 2$. Since $\frac{\phi^{\prime \prime}}{\left(a^{2}-A^{2}\right)}$ is positive definite, (3.35) follows after integrating (3.39) over $R \times(0, T)$ and applying the CauchySchwartz inequality and (3.34).

Lemma 3.3. There exist positive constants $\epsilon_{2}$ and $C$ such that if $E \leq \epsilon_{2}$ and $0 \leq t \leq T$, then

$$
\begin{aligned}
& \left\|\boldsymbol{f}_{x}(t) \#^{2}+\int_{0}^{t}\right\| \boldsymbol{f}_{x}(\tau) \|^{2} d \tau \\
& \leq\left\|\boldsymbol{f}_{x}(0) \#^{2}+C \int_{0}^{t}\right\| \nu-A \mu \|^{2} \\
& +C \int_{0}^{t} f\left(\left|\boldsymbol{v}^{(1)}\right|^{2}|\boldsymbol{f}|^{2}+|\boldsymbol{f}|^{6}+\left|\boldsymbol{v}_{x}^{(1)}\right|^{2}+\left|\boldsymbol{v}_{x x}^{(1)}\right|^{2}+\left|\boldsymbol{f}_{x}\right|^{2}\left|\boldsymbol{v}^{(1)}\right|\right) d x d \tau+C \alpha
\end{aligned}
$$

Proof. We first derive the equation for $\boldsymbol{f}_{x}$ by differentiating (2.17) with respect to $x$,

$$
\begin{aligned}
\boldsymbol{f}_{x t}+\boldsymbol{V} \boldsymbol{f}_{x x} & =-\left(\begin{array}{c}
O\left(\boldsymbol{v}_{x x}^{(1)}\right) \\
\boldsymbol{\nu}_{x}-\boldsymbol{A}_{1}\left(\boldsymbol{u}_{x}^{(0)}+\boldsymbol{\mu}_{x}\right)+\boldsymbol{A}_{0} \boldsymbol{u}_{x}^{(0)}+O\left(\boldsymbol{v}_{x x}^{(1)}\right) \\
O\left(\boldsymbol{v}_{x x}^{(1)}\right) \\
=-\left(\left|\boldsymbol{v} \boldsymbol{v}^{(1)}\right||\boldsymbol{f}|+|\boldsymbol{f}|\left|\boldsymbol{f}_{x}\right|+\left|\boldsymbol{v}_{x x}^{(1)}\right|\right)
\end{array}\right),
\end{aligned}
$$


where $\boldsymbol{A}_{0}=\mathcal{F}^{\prime}\left(\boldsymbol{u}^{(0)}\right)$ and $\boldsymbol{A}_{1}=\mathcal{F}^{\prime}\left(\boldsymbol{u}^{(0)}+\boldsymbol{\mu}\right)$. Next we multiply (3.46) from by

$$
f_{x}^{T}\left(\begin{array}{cc}
a^{2} \phi_{0}^{\prime \prime} & -\phi_{0}^{\prime \prime} A_{0} \\
-\phi_{0}^{\prime \prime} A_{0} & \phi_{0}^{\prime \prime}
\end{array}\right)
$$

to get

$$
\begin{aligned}
& \mathcal{E}_{0}\left[\boldsymbol{f}_{x}\right]_{t}+\mathcal{J}_{0}\left[\boldsymbol{f}_{x}\right]_{x} \\
= & -O(1)\left|\boldsymbol{\nu}_{x}-\boldsymbol{A}_{0} \boldsymbol{\mu}_{x}\right|^{2} \\
& +O(1)\left(\left|a^{2} \boldsymbol{\mu}_{x}-\boldsymbol{A}_{0} \boldsymbol{\nu}_{x}\right|\left|\boldsymbol{v}_{x x}^{(1)}\right|+\mid \boldsymbol{\nu}_{x}\right. \\
& \left.\quad-\left.\boldsymbol{A}_{0} \boldsymbol{\mu}_{x}\left|\left(\left|\boldsymbol{v}^{(1)}\right||\boldsymbol{f}|+|\boldsymbol{f}|\left|\boldsymbol{f}_{x}\right|+\left|\boldsymbol{v}_{x x}^{(1)}\right|\right)+\right| \boldsymbol{f}_{x}\right|^{2}\left|\boldsymbol{v}^{(1)}\right|\right)
\end{aligned}
$$

where

$$
\begin{gathered}
\phi_{0}^{\prime \prime}=\phi^{\prime \prime}\left(\boldsymbol{u}^{(0)}\right) \\
\mathcal{E}_{0}\left[\boldsymbol{f}_{x}\right]=\boldsymbol{f}_{x}^{T}\left(\begin{array}{cc}
a^{2} \phi_{0}^{\prime \prime} & -\phi_{0}^{\prime \prime} \boldsymbol{A}_{0} \\
-\phi_{0}^{\prime \prime} \boldsymbol{A}_{0} & \phi_{0}^{\prime \prime}
\end{array}\right) \boldsymbol{f}_{x} \\
\mathcal{J}_{0}\left[\boldsymbol{f}_{x}\right]=\boldsymbol{f}_{x}^{T}\left(\begin{array}{cc}
a^{2} \psi_{0}^{\prime \prime} & -\psi_{0}^{\prime \prime} \boldsymbol{A}_{0} \\
-\psi_{0}^{\prime \prime} \boldsymbol{A}_{0} & \psi_{0}^{\prime \prime}
\end{array}\right) \boldsymbol{f}_{x}
\end{gathered}
$$

and we have used the fact that $\phi_{0}^{\prime \prime} A_{0}$ is a symmetric matrix and

$$
\frac{\partial}{\partial x}\left(\begin{array}{cc}
a^{2} \phi_{0}^{\prime \prime} & -\phi_{0}^{\prime \prime} \boldsymbol{A}_{0} \\
-\phi_{0}^{\prime \prime} \boldsymbol{A}_{0} & \phi_{0}^{\prime \prime}
\end{array}\right)=O\left(\boldsymbol{v}^{(1)}\right) .
$$

We then integrate (3.48) over $R \times[0, t]$ to get, for $E$ sufficiently small and any $n$ there exists a $C>0$ such that

$$
\begin{aligned}
& \left\|f_{x}(t) \#^{2}+\int_{0}^{t}\right\| \nu_{x}-A_{0} \mu_{x} \#^{2} d \tau \\
\leq & \| f_{x}(0) \mathbb{H}^{2}+\int_{0}^{t} f m\left|a^{2} \mu_{x}-A_{0} \nu_{x}\right|^{2} \\
& +C\left(\left|\boldsymbol{v}^{(1)}\right|^{2}|\boldsymbol{f}|^{2}+\left|\boldsymbol{v}_{x x}^{(1)}\right|^{2}+\left|\boldsymbol{f}_{x}\right|^{2}\left|\boldsymbol{v}^{(1)}\right|\right) d x d \tau+C \alpha
\end{aligned}
$$

where we have used (3.34).

We now proceed to estimate $a^{2} \boldsymbol{\mu}_{x}-\boldsymbol{A}_{0} \boldsymbol{\nu}_{x}$.

First, let us rewrite (2.17) as

$$
\begin{aligned}
& \mu_{t}+\nu_{x}=\boldsymbol{v}_{x}^{(1)} \\
& \boldsymbol{\nu}_{t}+a^{2} \nu_{x}=-(\boldsymbol{\nu}-\boldsymbol{A} \boldsymbol{\mu})+O\left(|\boldsymbol{\mu}|^{3}\right)+O\left(\boldsymbol{v}_{x}^{(1)}\right)
\end{aligned}
$$

We now multiply the first equation of (3.50) by $-\boldsymbol{A}_{0}$ from the left and add it tc second:

$$
\left(\boldsymbol{\nu}_{t}-\boldsymbol{A}_{0} \boldsymbol{\mu}_{t}\right)+\left(a^{2} \boldsymbol{\mu}_{x}-\boldsymbol{A}_{0} \nu_{x}\right)=-(\boldsymbol{\nu}-\boldsymbol{A} \boldsymbol{\mu})+O\left(|\boldsymbol{\mu}|^{3}\right)+O\left(\boldsymbol{v}_{x}^{(1)}\right)
$$

so

$$
\begin{aligned}
& \left|a^{2} \boldsymbol{\mu}_{x}-\boldsymbol{A}_{0} \boldsymbol{\nu}_{x}\right|^{2} \\
= & -\left(\boldsymbol{\nu}_{t}-\boldsymbol{A}_{0} \boldsymbol{\mu}_{t}\right)^{T}\left(a^{2} \boldsymbol{\mu}_{x}-\boldsymbol{A}_{0} \boldsymbol{\nu}_{x}\right) \\
& -\left(\boldsymbol{\nu}-\boldsymbol{A} \boldsymbol{\mu}+O\left(|\boldsymbol{\mu}|^{3}\right)+O\left(\boldsymbol{v}_{x}^{(1)}\right)\right)^{T}\left(a^{2} \boldsymbol{\mu}_{x}-\boldsymbol{A}_{0} \boldsymbol{\nu}_{x}\right) .
\end{aligned}
$$


The second term on the right hand side of (3.52) is bounded by

$$
\begin{gathered}
-\left(\boldsymbol{\nu}-\boldsymbol{A} \boldsymbol{\mu}+O\left(|\boldsymbol{\mu}|^{3}\right)+O\left(\boldsymbol{v}_{x}^{(1)}\right)\right)^{T}\left(a^{2} \boldsymbol{\mu}_{x}-\boldsymbol{A}_{0} \nu_{x}\right) \\
\leq \quad \frac{1}{4}\left|a^{2} \boldsymbol{\mu}_{x}-\boldsymbol{A}_{0} \boldsymbol{\nu}_{x}\right|^{2}+C\left(|\boldsymbol{\nu}-\boldsymbol{A} \boldsymbol{\mu}|^{2}+|\boldsymbol{\mu}|^{6}+\left|\boldsymbol{v}_{x}^{(1)}\right|^{2}\right)
\end{gathered}
$$

while the first term is bounded by

$$
\begin{array}{ll} 
& \left(\left(\boldsymbol{\nu}-\boldsymbol{A}_{0} \boldsymbol{\mu}\right)_{t}+O\left(\left|\boldsymbol{v}^{(1)}\right||\boldsymbol{f}|\right)\right)^{T}\left(\left(a^{2} \boldsymbol{\mu}-\boldsymbol{A}_{0} \boldsymbol{\nu}\right)_{x}+O\left(\left|\boldsymbol{v}^{(1)}\right||\boldsymbol{f}|\right)\right) \\
\leq & \left(\boldsymbol{\nu}-\boldsymbol{A}_{0} \boldsymbol{\mu}\right)_{t}^{T}\left(a^{2} \boldsymbol{\mu}-\boldsymbol{A}_{0} \boldsymbol{\nu}\right)_{x} \\
& +C\left|\boldsymbol{v}^{(1)}\right||\boldsymbol{f}|\left(\left|\boldsymbol{\nu}_{t}-\boldsymbol{A}_{0} \boldsymbol{\mu}_{t}\right|+\left|a^{2} \boldsymbol{\mu}_{x}-\boldsymbol{A}_{0} \boldsymbol{\nu}_{x}\right|+O\left(\left|\boldsymbol{v}^{(1)} \| \boldsymbol{f}\right|\right)\right)
\end{array}
$$

Substituting (3.50) into the second term on the right hand side of (3.54), we can estimate it by

$$
\begin{aligned}
& C\left|\boldsymbol{v}^{(1)}\right||\boldsymbol{f}|\left(\left|\boldsymbol{\nu}_{t}-\boldsymbol{A}_{0} \boldsymbol{\mu}_{t}\right|+\left|a^{2} \boldsymbol{\mu}_{x}-\boldsymbol{A}_{0} \boldsymbol{\nu}_{x}\right|+\left|\boldsymbol{v}^{(1)}\right||\boldsymbol{f}|\right) \\
\leq & C\left|\boldsymbol{v}^{(1)}\right||\boldsymbol{f}|\left(2\left|a^{2} \boldsymbol{\mu}_{x}-\boldsymbol{A}_{0} \boldsymbol{\nu}_{x}\right|+|\boldsymbol{\nu}-\boldsymbol{A} \boldsymbol{\mu}|+|\boldsymbol{\mu}|^{3}+\left|\boldsymbol{v}_{x}^{(1)}\right|+\left|\boldsymbol{v}^{(1)}\right||\boldsymbol{f}|\right) \\
\leq & \frac{1}{4}\left|a^{2} \boldsymbol{\mu}_{x}-\boldsymbol{A}_{0} \boldsymbol{\nu}_{x}\right|^{2}+C\left(\left|\boldsymbol{v}^{(1)}\right|^{2}|\boldsymbol{f}|^{2}+|\boldsymbol{\nu}-\boldsymbol{A} \boldsymbol{\mu}|^{2}+|\boldsymbol{\mu}|^{6}+\left|\boldsymbol{v}_{x}^{(1)}\right|^{2}\right)
\end{aligned}
$$

while

$$
\begin{aligned}
& \left(\boldsymbol{\nu}-\boldsymbol{A}_{0} \boldsymbol{\mu}\right)_{t}^{T}\left(a^{2} \boldsymbol{\mu}-\boldsymbol{A}_{0} \boldsymbol{\nu}\right)_{x} \\
= & \left\{\left(\boldsymbol{\nu}-\boldsymbol{A}_{0} \boldsymbol{\mu}\right)^{T}\left(a^{2} \boldsymbol{\mu}-\boldsymbol{A}_{0} \boldsymbol{\nu}\right)_{x}\right\}_{t} \\
& -\left\{\left(\boldsymbol{\nu}-\boldsymbol{A}_{0} \boldsymbol{\mu}\right)^{T}\left(a^{2} \boldsymbol{\mu}-\boldsymbol{A}_{0} \boldsymbol{\nu}\right)_{t}\right\}_{x}+\left(\boldsymbol{\nu}-\boldsymbol{A}_{0} \boldsymbol{\mu}\right)_{x}^{T}\left(a^{2} \boldsymbol{\mu}-\boldsymbol{A}_{0} \boldsymbol{\nu}\right)_{t}
\end{aligned}
$$

The last term in the right hand side of (3.56) can be similarly treated as in (3.55):

$$
\left(\boldsymbol{\nu}-\boldsymbol{A}_{0} \boldsymbol{\mu}\right)_{x}^{T}\left(a^{2} \boldsymbol{\mu}-\boldsymbol{A}_{0} \boldsymbol{\nu}\right)_{t} \leq C\left(\left|\nu_{x}-\boldsymbol{A}_{0} \boldsymbol{\mu}_{x}\right|^{2}+\left|\boldsymbol{v}^{(1)}\right|^{2}|\boldsymbol{f}|^{2}\right) .
$$

After integrating over $R \times(0, t)$ and applying (3.34), we have

$$
\begin{aligned}
& \int_{0}^{t} \| a^{2} \boldsymbol{\mu}_{x}-\boldsymbol{A}_{0} \boldsymbol{\nu}_{x} \#^{2} d \tau \leq \\
& C\left\{\int_{0}^{t}\|\boldsymbol{\nu}-\boldsymbol{A} \boldsymbol{\mu}\|^{2} d \tau+\int_{0}^{t} \| \boldsymbol{\nu}_{x}-\boldsymbol{A}_{0} \boldsymbol{\mu}_{x} \#^{2} d \tau+\int_{0}^{t} \int\left(\left|\boldsymbol{v}^{(1)}\right|^{2}|\boldsymbol{f}|^{2}+|\boldsymbol{f}|^{6}+\left|\boldsymbol{v}_{x}^{(1)}\right|^{2}\right) d x d \tau\right\} \\
& +C \alpha
\end{aligned}
$$

Since

$$
c_{1}\left(\left|\nu_{x}\right|^{2}+\left|\boldsymbol{\mu}_{x}\right|^{2}\right) \leq\left|a^{2} \boldsymbol{\mu}_{x}-\boldsymbol{A}_{0} \nu_{x}\right|^{2}+\left|\nu_{x}-\boldsymbol{A}_{0} \boldsymbol{\mu}_{x}\right|^{2} \leq c_{2}\left(\left|\nu_{x}\right|^{2}+\left|\boldsymbol{\mu}_{x}\right|^{2}\right)
$$

for sufficiently large $a$, we conclude with (3.45) from (3.49) and (3.57).

Combining Lemma 3.2 and Lemma 3.3 with suitably chosen $m, \alpha$ and $E$, we have for $0 \leq t \leq T$,

$$
\begin{aligned}
& \left\|\boldsymbol{f}(t) \#_{1}^{2}+\int_{0}^{t}\right\| \boldsymbol{f}_{x} \#^{2} d \tau \\
\leq & \| \boldsymbol{f}(0) H_{1}^{2} \\
& +C_{1} \int_{0}^{t} f\left(\left|\boldsymbol{v}^{(1)}\right|^{4}+\left|\boldsymbol{v}_{x}^{(1)}\right|^{2}+|\boldsymbol{f}|\left|\boldsymbol{v}^{(1)}\right|+|\boldsymbol{f}|^{6}+\left|\boldsymbol{v}_{x x}^{(1)}\right|^{2}+\left|\boldsymbol{f}_{x}\right|^{2}\left|\boldsymbol{v}^{(1)}\right|\right) d x d \tau+C \alpha
\end{aligned}
$$


Finally, we can estimate the right hand side of (3.60) by

LEMMA 3.4. There exist a positive constant $C$ such that if $E<\epsilon_{2}$ and $0 \leq t \leq T$, then

(3.61) the right hand side of (3.60) $\leq C\left(\left\|f(0) \#_{1}^{2}+\alpha^{1 / 6}+(E+\alpha) \int_{0}^{t}\right\| f_{x} \#^{2} d \tau\right)$.

Proof. We estimate each term by virtue of Lemma 2.2 and the Sobolev inequality (2.21) as follows:

$$
\begin{aligned}
& \int^{t} f\left|\boldsymbol{v}^{(1)}\right|^{4} d x d \tau \leq C \int_{0}^{t}\left\|\boldsymbol{v}^{(1)}\right\|_{L^{\infty}}^{2}\left\|\boldsymbol{v}^{(1)}\right\|^{2} d \tau \leq C \alpha \\
& \int_{0}^{t} f\left|\boldsymbol{v}_{x}^{(1)}\right|^{2}+\left|\boldsymbol{v}_{x x}^{(1)}\right|^{2} d x d \tau \leq C \alpha^{\frac{1}{4}} \\
& \int_{0}^{t} f|\boldsymbol{f}|\left|\boldsymbol{v}_{x}^{(1)}\right| d x d \tau \leq C \int_{0}^{t}\left(\|\boldsymbol{f}\|^{\frac{1}{2}}\left\|\boldsymbol{f}_{x}\right\|^{\frac{1}{2}}+\alpha e^{-C \tau}\right)\left\|\boldsymbol{v}_{x}^{(1)}\right\|_{L^{1}} d \tau \\
& \leq C \int_{0}^{t}\left(E^{2}\left\|\boldsymbol{f}_{x}\right\|^{2}+\left\|\boldsymbol{v}_{x}^{(1)}\right\|_{L^{1}}^{\frac{4}{3}}\right) d \tau+C \alpha \\
& \leq C \int_{0}^{t}\left(E^{2} \| \boldsymbol{f}_{x} \#^{2}+\alpha^{\frac{1}{6}}(1+\tau)^{\frac{-7}{6}}\right) d \tau+C \alpha \\
& \leq C E \int_{0}^{t}\left\|\boldsymbol{f}_{x}\right\|^{2} d \tau+C \alpha^{\frac{1}{6}} \\
& \int_{0}^{t} f|\boldsymbol{f}|^{6} d x d \tau \leq \int_{0}^{t}\|f\|^{4}\left\|f_{x} \#^{2} d \tau+C \alpha \leq E \int_{0}^{t}\right\| f_{x} \#^{2} d \tau+C \alpha \\
& \int_{0}^{t} f\left|\boldsymbol{f}_{x}\right|^{2}\left|\boldsymbol{v}^{(1)}\right| d x d \tau \leq \alpha \int_{0}^{t} \| \boldsymbol{f}_{x} \mathbb{H}^{2} d \tau \\
& \int_{0}^{t} f|\boldsymbol{f}|^{2}\left|\boldsymbol{v}^{(1)}\right| d x d \tau \leq C \int_{0}^{t}\|\boldsymbol{f}\|^{\frac{3}{2}}\left\|\boldsymbol{f}_{x}\right\|^{\frac{1}{2}}\left\|\boldsymbol{v}^{(1)}\right\|_{L^{\infty}} d \tau+C \alpha \\
& \leq C \int_{0}^{t}\left(E\left\|\boldsymbol{f}_{x}\right\|^{2}+\left\|\boldsymbol{v}^{(1)}\right\|_{L^{\infty}}^{\frac{4}{3}}\right) d \tau+C \alpha \\
& \leq C E \int_{0}^{t} \| \boldsymbol{f}_{x} \#^{2} d \tau+C \alpha^{\frac{1}{6}}
\end{aligned}
$$


[2] G.-Q Chen, C. Levermore AND T.-P. LiU, Hyperbolic conservation laws with stiff relaxation terms and entropy, Comm. Pure Appl. Math., 47 (1994), pp. 787-830.

[3] Gosse, L. And Tzavaras, A. E., Convergence of relaxation schemes to the equation of elastodynamics, Arch. Rat. Mech. and Anal. 121 (1992), pp. 235-265.

[4] Jin, S., Katsulakis, M. A. And XIN, Z., Relaxation schemes for curvature dependent front propagation, Comm. Pure. Appl. Math. 52 (1999) pp. 1587-1615.

[5] JIN, S. AND XIN, Z., The relaxation schemes for systems of conservation laws in arbitrary space dimensions, Comm. Pure Appl. Math., 48(3)(1995), pp. 235-276.

[6] JIN, S. AND XIN, Z., Numerical passage from systems of conservation laws to HamiltonJacobi equations, SIAM J. Numer. Anal. 35 (1998), pp.2385-2404.

[7] T.-P. LiU, Hyperbolic conservation laws with relaxation, Comm. Math. Phys. 108 (1987), pp. 153-175.

[8] T. LUO, Asymptotic stability of planar rarefaction waves for the relaxation approximation of conservation laws in several dimensions, J. Diff. Equa., 133 (1997), pp. 255-279.

[9] LUO, T. AND XIN, Z., Nonlinear stability of shock fronts for a relaxation system in several space dimensions, J. Diff. Equa., 139 (1997), pp. 365-408.

[10] Matsumura, A. And Nishihara, K., Asymptotics toward the rarefaction waves of the solutions of a one-dimensional model system for compressible viscous gas, Japan J. Appl. Math, 3(1)(1986), pp. 1-13.

[11] Natalini, R., Convergence to equilibrium for the relaxation approximations of conservation laws, Comm. Pure Appl. Math., 49(8)(1996), pp. 795-823.

[12] RAUCH, J. AND REED, M., Jump discontinuities of semilinear, strictly hyperbolic systems in two variables: creation and propagation, Comm. Math. Phys, 81(2)(1981), pp. 203-227.

[13] D. SerRe, Relaxation semi-lineaire des systemes de lois de conservation, Ann. Inst. H. Poincar Anal. Non Lineaire 17 (2000), pp. 169-192.

[14] J. SMOller, Shock Waves and Reaction-Diffusion Equations, New York, SpringerVerlag, 1983.

[15] Teng, Z. H., Firsr order $L^{1}$ for the relaxation approximation to conservation laws, Comm. Pure Appl. Math., 51 (1998), pp. 857-895.

[16] Tzavaras, A. E., Materials with internal variables and relaxation to conservation laws, Arch. Ration. Mech. Anal. 146 (1999) 129-155.

[17] TAdmor, E. AND TANg, T., Pointwise error estimate for relaxation approximations to conservation laws, SIAM J. Math. Anal., 32 (2000) 87, pp. 870-886. 
W. C. WANG 